\title{
Alterstice
}

Revue internationale de la recherche interculturelle

International Journal of Intercultural Research

Revista International de la Investigacion Intercultural

\section{Le rôle du père dans le développement socio-affectif et cognitif des enfants en contexte migratoire}

\section{Jean Ramdé}

Volume 5, numéro 1, 2015

Engagement paternel et migration

URI : https://id.erudit.org/iderudit/1077303ar

DOI : https://doi.org/10.7202/1077303ar

Aller au sommaire du numéro

Éditeur(s)

Alterstice

ISSN

1923-919X (numérique)

Découvrir la revue

Citer ce document

Ramdé, J. (2015). Le rôle du père dans le développement socio-affectif et cognitif des enfants en contexte migratoire. Alterstice, 5(1), 3-6.

https://doi.org/10.7202/1077303ar

\section{Résumé de l'article}

Les recherches sur la relation père-enfant et le développement de l'enfant sont très peu nombreuses comparativement à celles sur la relation mère-enfant. Les rares études sur le rôle du père sur le développement de l'enfant montrent que le père contribue de façon significative au développement socio-affectif et cognitif de l'enfant. Ce constat a permis l'accroissement et la diversification des recherches sur l'engagement paternel. Toutefois, l'engagement paternel en contexte migratoire, surtout dans l'espace francophone, est très peu connu. Ce numéro thématique tente de faire un premier pas pour combler cette lacune, en offrant un espace de réflexion et de partage des connaissances théoriques, cliniques et empiriques sur le sujet. Cette réflexion touche à la fois à l'engagement des pères, au vécu des pères migrants et à la transmission des valeurs. 
INTRODUCTION THÉMATIQUE

\title{
Le rôle du père dans le développement socio-affectif et cognitif des enfants en contexte migratoire
}

\author{
Jean Ramdé ${ }^{1}$
}

\section{Résumé}

Les recherches sur la relation père-enfant et le développement de l'enfant sont très peu nombreuses comparativement à celles sur la relation mère-enfant. Les rares études sur le rôle du père sur le développement de l'enfant montrent que le père contribue de façon significative au développement socio-affectif et cognitif de l'enfant. Ce constat a permis l'accroissement et la diversification des recherches sur l'engagement paternel. Toutefois, l'engagement paternel en contexte migratoire, surtout dans l'espace francophone, est très peu connu. Ce numéro thématique tente de faire un premier pas pour combler cette lacune, en offrant un espace de réflexion et de partage des connaissances théoriques, cliniques et empiriques sur le sujet. Cette réflexion touche à la fois à l'engagement des pères, au vécu des pères migrants et à la transmission des valeurs.

\section{Rattachement de l'auteur}

${ }^{1}$ Faculté des sciences de l'éducation, Université Laval, Québec, Canada

\section{Correspondance}

jean.ramde@fse.ulaval.ca

\section{Mots clés}

engagement paternel, pères migrants, relation père-enfant, rôle du père

\section{Pour citer cet article}

Ramdé, J. (2015). Le rôle du père dans le développement socio-affectif et cognitif des enfants en contexte migratoire. Alterstice, 5(1), 3-6. 
Si la relation mère-enfant a été abondamment étudiée, celle qui lie le père à ses enfants reste encore en partie méconnue (Korff-Sausse et Sacco, 2012). Toutefois, depuis 20 ans, le père retient de plus en plus l'attention des chercheurs. La très grande majorité des études sur le père sont été consacrées à l'engagement paternel ou father involvement. Lamb (1981) définit cet engagement paternel comme (1) le temps consacré par le père aux interactions directes avec l'enfant, de nature ludique, affective et sociale, (2) la disponibilité du père à l'enfant et (3) la responsabilité assumée par le père dans le soin et l'éducation des enfants et dans le partage des tâches parentales. De manière générale, deux formes d'engagement paternel s'observent dans les interactions pèreenfant: d'une part, un engagement direct caractérisé par les soins, l'autorité et le jeu et, d'autre part, un engagement indirect caractérisé par le rôle de pourvoyeur envers l'enfant et la mère. Selon les cultures, l'une ou l'autre forme sera davantage favorisée.

Les domaines couverts par la recherche consacrée à la question du rôle du père dans le développement socioaffectif et cognitif des enfants touchent, entre autres, l'impact de l'engagement paternel dans la différenciation sexuelle (Lamb et Tamis-LeMonda, 2004; Le Camus, 2005; Parke, 1996), l'absence du père et le développement de l'enfant (Bögels et Phares, 2008; Choi et Jackson, 2011; Flouri et Malmberg, 2012), la fréquence et la qualité des interactions entre le père et ses enfants (Amato et Rivera, 1999; Livingston et Parker, 2011; Paquette, 2004) et le soutien indirect du père (Lamb, 2010).

Lamb (1997) estime que l'état actuel des connaissances sur le père et le développement de l'enfant fournit des preuves substantielles que les nourrissons peuvent former des attachements avec aussi bien les mères que les pères et à peu près au même moment, à savoir pendant la première année de vie. De plus, les réactions d'attachement et l'angoisse de la séparation existent autant pour le père que pour la mère (Lamb, 2004; Le Camus, 2000). Le Camusarrive à la conclusion que les pères y figurent aussi comme objet d'attachement, au même titre que les mères (Le Camus, 2000, 2005). Les pères peuvent ainsi être considérés comme des partenaires du bébé émotionnellement compétents.

De façon générale, l'engagement paternel a des effets certains sur le développement cognitif et socio-affectif de l'enfant (Lamb, 2004). Sur le plan cognitif, ces effets sont perceptibles dès l'âge de six mois. Par exemple, le jeune enfant qui a bénéficié de cet engagement obtient un score plus élevé sur les échelles du développement, un niveau de fonctionnement cognitif plus élevé, réussit mieux à résoudre les problèmes et a un QI plus élevé. De plus, la scolarité des enfants dont le père est activement engagé est plus linéaire. Les garçons, en particulier, ont de ce fait une meilleure carrière professionnelle, notamment parce que leur désir de réussite ne serait pas entravé par une trop grande fermeture (autoritarisme) ou par trop d'ouverture (coopératisme).

Au niveau socio-affectif, l'engagement paternel permet aux enfants d'être plus confiants dans leurs capacités, d'être plus responsables et d'échanger plus facilement avec les adultes et les pairs dès l'âge préscolaire. L'engagement paternel a aussi été associé à une satisfaction globale dans la vie chez l'enfant et à une diminution des risques de dépression, de détresse émotionnelle, d'expression d'émotions négatives comme la peur et le sentiment de culpabilité, de problèmes comportementaux et de détresse psychologique. Le paradigme de l'engagement et du rôle paternels devient de ce fait incontournable dans l'étude du développement socio-affectif et cognitif de l'enfant, d'autant plus que le père peut y figurer à titre de compagnon, donneur de soins, protecteur, modèle, guide moral, instructeur et pourvoyeur (Lamb, 1997).

Par ailleurs, depuis quelques années, le rôle du père dans divers contextes culturels retient l'attention des chercheurs (Hewlett, 2000, 2004; Roopnarine, 2002). Cet intérêt s'est notamment matérialisé par la publication d'un ouvrage collectif où les auteurs passent en revue les rôles des pères en Asie, Afrique, Amérique, Europe et Australie et qui présente la recherche sur la paternité dans une perspective interculturelle et apporte aux études contemporaines une contribution d'une importance capitale (Shwalb, Shwalb et Lamb, 2013). Toutefois, la question de la paternité en contexte migratoire n'y est pas abordée.

De fait, malgré les bienfaits de l'engagement paternel décrits plus haut, très peu de publications dans l'espace francophone portent sur l'engagement paternel en contexte migratoire (Battaglini, Gravel, Poulin, Fournier et Brodeur, 2002; Dyke et Saucier, 2000; Jain et Belsky, 1997; Strier et Roer-Strier 2010). Selon Dyke et Saucier (2000), 
l'immigration vient ébranler les zones d'influence sur les plans à la fois individuel (effritement des repères), relationnel (difficultés conjugales) et contextuel (absence de soutien social) de l'engagement paternel. Toujours selon ces auteurs, le fait de devoir s'adapter et d'être au cœur d'un processus de changement fondamental, la perte d'illusions et le dur contact avec la réalité pourraient contribuer à réduire, entre autres, la disponibilité émotionnelle d'un homme et sa capacité à se sentir concerné par les affaires familiales. Par ailleurs, certains migrants viennent de pays où l'engagement paternel est indirect.

Ce numéro thématique amorce un premier pas pour combler les lacunes dans la recherche sur l'engagement paternel en contexte migratoire, en offrant un espace de réflexion et de partage des connaissances théoriques et empiriques sur le sujet. Cette réflexion touche à la fois au rôle des pères, au vécu des pères migrants et à la transmission des valeurs. Les articles proposés ici font état d'études dans des contextes fort variés mais avec des points communs, susceptibles d'être autant de pistes de recherches futures : nous espérons ainsi susciter un intérêt pour la recherche sur les facteurs qui influencent l'engagement paternel en contexte migratoire, les types d'engagement paternel, l'acculturation et l'engagement paternel, les conséquences de l'engagement paternel des immigrants, l'intervention auprès des pères immigrants, etc.

L'organisation du numéro permet de faire un voyage. Nous amorçons la lecture par un article pouvant sembler de prime abord un peu décalé : il y est question du père dans un contexte culturel habituellement considéré comme fort différent de celui de l'Occident, le Japon. Ce choix est motivé par une volonté de décentrer le lecteur afin qu'il prenne conscience des différences dans la conception des rôles du père. Nous explorons ensuite le vécu migratoire dans différents contextes nationaux (Québec, France et Belgique) et nous terminons par une étude sur les pères de seconde génération (issus de pères immigrants) en France.

Valérie Harvey et Diane-Gabrielle Tremblay traitent dans le premier article, "Le père japonais dans une société ultramobile de célibataires ", du peu de mesures mises en place par l'État japonais pour encourager la parentalité. Cette situation contribue à faire du Japon une société de célibataires. Le texte en offre la démonstration en traitant des congés parentaux et de la division du travail dans un ménage japonais et des effets de l'ensemble sur la famille.

Dans le deuxième article, "Regards croisés sur les enjeux rencontrés par les pères immigrants au Québec ", Francine de Montigny, Normand Brodeur, Christine Gervais, Denise Pangop et Assumpta Ndengeyingoma font ressortir, à l'aide d'un modèle d'analyse écosystémique, les convergences entre les différentes études sur les pères immigrants au Québec, ce qui permet de dégager des pistes pour l'amélioration des services offerts dans les milieux institutionnels et communautaires.

Le troisième article, de Dimitri Cauchie, M. Bruyninckx et Ornella Cappalonga s'intitule « Vécu de la paternité et implication dans les soins périnataux: rencontres avec des hommes africains émigrés en Belgique ». Pour les besoins de cette étude, les auteurs ont rencontré deux fois les participants, une première fois durant la grossesse et une seconde environ deux semaines après la naissance. Les participants ne partagent pas tous la même opinion quant à une distinction genrée des rôles éducatifs parentaux et, parmi ceux témoignant de changements comportementaux, certains ne les maintiendraient pas dans leur contexte culturel d'origine.

Jérôme Gidoin rend compte dans le quatrième article, "La transmission père-fils des valeurs filiales vietnamiennes à partir du bouddhisme en France ", d'une enquête ethnologique portant sur la réinterprétation du culte des ancêtres des Vietnamiens en France. De plus en plus de familles vietnamiennes, par commodité, délèguent leur culte des ancêtres domestique à des moines. L'auteur s'interroge alors sur l'engagement des pères: en quoi peuvent-ils être considérés comme les acteurs d'un processus d'ethnicisation faisant la jonction entre le passé et le présent? Peut-on parler d'une redéfinition de l'identité paternelle via le référent bouddhiste en France?

Myriam Kettani, discute, dans le cinquième et dernier article, "L'engagement paternel des pères d'origine maghrébine en France ", de la parentalité des jeunes d'origine maghrébine issus de l'immigration. Ce type de paternité est encore mal connu et peu étudié. L'article propose donc d'explorer les expériences des pères d'origine maghrébine en France et de retracer à partir de la littérature existante le parcours intergénérationnel qui va des pères immigrés aux pères issus de l'immigration. 


\section{Références bibliographiques}

Amato, P. et Gilbreth, J. (1999). Non-resident fathers and children's well-being: A metaanalysis. Journal of Marriage and Family, 61(3), 557-573.

Amato, P. et Rivera, F. (1999). Paternal involvement and children's behavior problems. Journal of Marriage and Family, 61(2), 375-384.

Battaglini, A., Gravel, S., Poulin, C., Fournier, M. et Brodeur, J.-M. (2002). Migration et paternité ou réinventer la paternité. NPS, 15(1), 165-179.

Bögels, S. et Phares, V. (2008). The role of the father in the development, prevention and treatment of childhood anxiety disorders: A review and new model. Clinical Psychology Review, 28, 539-558.

Choi, J. et Jackson, A. (2011). Fathers' involvement and childbehavior problems in poorAfricanAmerican singlemother families. Children and YouthServices Review, 33, 698-704.

Dyke, N. et Saucier, J.-F. (2000). Cultures et paternités : impacts de l'immigration. Montréal : Éditions Saint-Martin.

Flouri, E. et Malmberg, L. (2012). Fathers' involvement and preschool children's behavior in stable single-mother families. Children and Youth Services Review, 34(7), 1237-1242.

Jain, A. et Belsky, J. (1997). Fathering and acculturation: a atudy of immigrant Indian families with young children. Journal of Marriage and the Family, 59, 873-883.

Hewlett, B. (2000). Culture, history, and sex: anthropological contributions to conceptualizing father involvement. Marriage and family review, 29(2/3), 59-73.

Hewlett, B. (2004). Fathers in forager, farmer, and pastoral cultures. Dans M. Lamb (dir.), The role of father in child development ( $4^{\mathrm{e}}$ éd.) (p. 182-195). Hoboken, NJ : Wiley.

Korff-Sausse, S. et Sacco, F. (2012). Être père aujourd'hui : un modèle à réinventer. Paris : In Press

Lamb, M. (1981). The role of the father in child development ( $2^{\mathrm{e}}$ éd.). New York : Wiley.

Lamb, M. (1997). The role of the father in child development ( $3^{\mathrm{e}}$ éd.). New York : Wiley.

Lamb, M. (2004). The role of the father in child development ( $4^{\mathrm{e}}$ éd.). Hoboken, NJ : Wiley .

Lamb, M. (2010). The role of the father in child development ( $5^{\mathrm{e}}$ éd.). Hoboken, NJ : Wiley.

Lamb, M. et Tamis-LeMonda, C. (2004). The role of the father: An introduction. Dans M. Lamb (dir.), The role of the father in child development ( $4^{\mathrm{e}}$ éd.) (p. 1-31). Hoboken, NJ : Wiley.

Le Camus, J. (2000). Le vrai rôle du père. Paris : Odile Jacob.

Le Camus, J. (2005). Comment être père aujourd'hui? Paris : Odile Jacob.

Livingston, G. et Parker, K. (2011). A tale of two fathers: More are active, but more are absent. Washington, DC : Pew research center. http://www.pewsocialtrends.org/files/2011/06/fathers-FINAL-report.pdf

Paquette, D. (2004). Theorizing the Father-Child Relationship: Mechanisms and Developmental Outcomes. Human Development, 47(4), 193-219.

Parke, R. (1996). Fatherhood. Cambridge : Harvard University Press.

Shwalb, D., Shwalb B. et Lamb M. (2013). Fathers in cultural context. New York : Routledge.

Strier, R. et Roer-Strier, D. (2010). Fatherhood in the context of immigration. Dans M. Lamb (dir.), The role of the father in child development ( $5^{\mathrm{e}}$ éd.) (p. 435-458). Hoboken, NJ : Wiley.

Roopnarine, L. (2002). Father involvement in english-speaking Caribbean families. Dans C. Tamis-LeMonda et N. Cabrera (dir.), Handbook of father Involvement: multidisciplinary perspectives (p. 249-277). Mahwah : LEA.

Alterstice - Revue Internationale de la Recherche Interculturelle, vol. 5, $n^{\circ} 1$ 